\title{
Health and Risks: Integrating Health into Disaster Risk Reduction, Risk Communication, and Building Resilient Communities
}

\author{
Emily Ying Yang Chan ${ }^{1,2} \cdot$ Peijun $\mathrm{Shi}^{3}$
}

Published online: 21 June 2017

(c) The Author(s) 2017. This article is an open access publication

In recent years, "health" has been recognized explicitly as both a determinant and an outcome of the human dimension of disaster risk reduction (DRR). Every disaster creates significant public health needs that can exceed local capacity to respond, resulting in excess mortality and morbidity. The European Union report on Science for Disaster Risk Management 2017: Knowing Better and Losing Less recommended that health sciences should be more involved in the community of disaster risk management to advance our understanding of outbreaks and pandemics and the health impacts of all hazards, and to improve data collection. Promoting systematic integration of health considerations into disaster risk reduction policies and plans and the inclusion of emergency and disaster risk management professionals in health strategies has become urgent and necessary. Furthermore, effective communication about health risks that aims to promote resilience of communities in all phases of the emergency process-

Emily Ying Yang Chan

emily.chan@cuhk.edu.hk

1 Collaborating Centre for Oxford University and CUHK for Disaster and Medical Humanitarian Response (CCOUC), The Jockey Club School of Public Health and Primary Care, The Chinese University of Hong Kong, Hong Kong, China

2 World Health Organization (WHO) Thematic Platform for Health Emergency and Disaster Risk Reduction Management (Health-EDRM) Research Group, The Chinese University of Hong Kong, Hong Kong, China

3 State Key Laboratory of Earth Surface Processes and Resource Ecology/Academy of Disaster Reduction and Emergency Management, Faculty of Geographical Science, Beijing Normal University, Beijing 100875, China prevention, preparedness, response, and recovery-is imperative.

Asia-Pacific is the world's most disaster-prone region. In addition to geophysical hazards, global climate change will increase the probability of extreme weather events, such as heat waves, droughts, wildfires, cyclones, and heavy precipitation that could cause floods and landslides. More people will be exposed to climate-related natural hazards and greater disaster risks might be experienced, especially in less developed countries. Certain population subgroups, such as the poor and marginalized, people with disabilities, women and children, and the elderly will bear the greatest burden of risk in all regions.

In this special section, six papers-related to evidence from health emergency and disaster risk reduction-share some of the recent research in the area of health in disaster risk science. Lo et al. discuss the latest research paradigm of Health Emergency and Disaster Risk Management (Health-EDRM) that encompasses DRR, emergency and disaster medicine, humanitarian response, community health, and health systems resilience. It highlights the intersections of health and DRR that have emerged in recent years as a field of critical inquiry as both outcomes and goals of DRR to ensure the implementation of the Sendai Framework for Disaster Risk Reduction 20152030. At the policy level, Maini et al. argue that although the Sendai Framework recognizes the strong connections between health and disasters and promotes the concept of health resilience throughout, one of the key research challenges is the lack of multidisciplinary, agreed indicators and definitions, which might hamper the implementation of the global agreement.

Disaster resilience depends on health risk profiling and community preparedness. Sakamoto's article examines 
how potential health risks are associated with water salinity level, especially related to climate change, and how selfreported diet salt intake might vary with sociodemographic characteristics in the coastal area of Southeastern Bangladesh. Sun et al. argue that DRR should be place- and ageresponsive, and their study shows how the concept and contents of DRR may be reformulated through applying a collaborative place audit (CPA) and social vulnerability index (SVI). In addition to disaster health risk profiling, a better strategy is required for disaster risk communication and indicating the potential challenges facing the vulnerable population during extreme weather events and climate change in the decades to come. Chan, Huang, et al.'s work in urban weather information acquisition patterns and their health significance during extreme cold weather in Hong Kong offers a case study to emphasize this issue. For the rural community, Chan, Guo, et al. share research evidence in the implementation of a bottom-up, field-based HealthEDRM program in remote, flood-prone ethnic minority rural China.

The studies in this special section show how research enquiry into health may be integrated into disaster risk reduction, risk communication, and the building of resilient communities. In the years to come, interdisciplinary collaboration research with the inclusion of health will be one of the new frontiers for the disaster risk research community to explore and address.

Open Access This article is distributed under the terms of the Creative Commons Attribution 4.0 International License (http://crea tivecommons.org/licenses/by/4.0/), which permits unrestricted use, distribution, and reproduction in any medium, provided you give appropriate credit to the original author(s) and the source, provide a link to the Creative Commons license, and indicate if changes were made. 